\title{
New Possibilities of Mathematical Modeling of Turbulent Transport Processes in Plasma
}

\author{
SKVORTSOVA Nina, BATANOV German, PETROV Alexander, PSHENICHNIKOV Anton, SARKSIAN Karen, \\ KHARCHEV Nikolai, BENING Vladimir ${ }^{1}$, KOROLEV Victor ${ }^{1}$, MARAVINA Tatiana ${ }^{1}$, NASYROV Farit ${ }^{2}$, \\ SANCHEZ Joaquin ${ }^{3}$, OHKUBO Kunizo ${ }^{4}$, SHIMOZUMA Takashi ${ }^{4}$, IDEI Hiroshi ${ }^{4}$, \\ YOSHIMURA Yasuo ${ }^{4}$ and KUBO $\mathrm{Shin}^{4}$ \\ General Physics Institute, Moscow, Russia \\ ${ }^{I}$ Moscow State University, Moscow, Russia \\ ${ }^{2}$ Ufa State Aviation Technical University, Ufa, Russia \\ ${ }^{3}$ EUROATOM-CIEMAT, Madrid, Spain \\ ${ }^{4}$ National Institute for Fusion Science, Toki 509-5292 Japan \\ (Received: 9 December 2003 / Accepted: 29 April 2004)
}

\begin{abstract}
Measurements in the LHD, L2-M, and TJ-II stellarators show that plasma density fluctuations have non-Gaussian distributions with heavy tails and sharper vertices. Non-Gaussian probability densities of stochastic plasma processes indicate non-Brownian character of the motion (diffusion) of particles. The role of rare events related to stochastic plasma processes with larger spatial and temporal scale becomes important. It is shown that the first-order differences of fluctuation samples are stochastic and their probability distribution is a mixture of Gaussians with different scales. Subordinated Lèvy process can be used to describe the turbulent transport process.
\end{abstract}

\section{Keywords:}

plasma turbulence, fluctuation, non-Gaussian process, Lèvy processes, stellarator, scattering technique

\section{Introduction}

Recently, low-frequency (LF) plasma turbulence in closed magnetic confinement systems attracted considerable interest of plasma physicists. The 2003 EPS conference demonstrated that experimental research of this kind is now being carried out in all of the existing tokamaks and stellarators: T-10, LHD, TJ-II, DII-D, JET, etc. (see [1], report nos. P-2.56, P-3.121, P-4.5, O-2.1A, and P-120). A reason for the increase of interest in LF turbulence is that many experimental facts directly indicate its influence on the macroscopic plasma parameters in closed magnetic confinement systems.

In many toroidal devices LF turbulence in core and edge plasmas is strong structural $[2,3]$. The term "strong structural turbulence" means that on the background of strong turbulence due to the onset of plasma instabilities, there exists an ensemble of the interacting stochastic plasma structures. Various types of stochastic structures (such as solitons, vortices and MHD structures) were observed experimentally in the edge plasma [4-6]. It was found that the strong structural turbulence existing in the edge plasma possesses some general properties irrespective of the turbulence source and the type of stochastic plasma structures. The turbulence of this kind is characterized by slowly decreasing

Corresponding author's e-mail: nina@fpl.gpi.ru autocorrelation functions with oscillating tails and waveletspectra show distinct harmonics. At the same time, the probability density function (PDF) of the time sample of magnitudes differs from a Gaussian distribution [7,8].

According to the traditional viewpoint on what goes on in plasma turbulence, the distributions of processes registered in experiments in stellarators should be normal. However, the statistical analysis of experimental data demonstrates that in practice this is far from being so. The obtained empirical distributions are usually far from being normal possessing densities with tails heavier and vertices sharper than those inherent in the normal law [7]. This means that the registered processes are far from being successfully modeled by the Brownian motion. Moreover, it was statistically made evident that these processes possess a kind of memory exhibiting the presence of long-range dependence of their values [8].

Recently, many alternative models were proposed for these processes. First of all, it should be mentioned that, following the ideology of Hurst and Mandelbrot [9], in many papers the authors investigated the possibility of application of the so-called fractional Brownian motion as an alternative to pure Brownian motion. We should even say that the fractional Brownian motion is a very popular model. Unlike Science and Nuclear Fusion Research 
pure Brownian motion, fractional Brownian motion has an indisputable advantage, which consists in that this process can successfully model the long-range dependence of the values of registered data (time series). However, we dare say that along with this advantage, fractional Brownian motion has a no less indisputable drawback, which consists in that all its finite-dimensional distributions remain Gaussian, contradicting to what is observed in practice. Therefore, alternative models have to be designed. Recently it was proposed to model plasma turbulence by the so-called subordinated Lèvy processes. Recall that a Lèvy process is a stochastic process with stationary independent first-order differences. A subordinated process is a stochastic process of the form $S(t)=X(M(t)), t \geq 0$, where $X(t)$ and $M(t)$ are stochastic processes such that $X(t)$ is measurable and the trajectories of the subordinator $M(t)$ start from the origin and do not decrease. In this setting the process $M(t)$ plays the role of random time. The application of subordinated Lèvy processes to the interpretation of the structural plasma turbulence was proposed by V. Korolev and was justified in the corresponding limit theorems, see $[10,11]$. Here we only mention two attractive properties of subordinated Lèvy processes, namely, their self-similarity and the representability of their finite-dimensional distributions as scale/location mixtures of Gaussian laws.

In the problems under discussion the intensity of processes substantially varies in time so that the notion of subordination seems to be very adequate. Thus, the amplitudes of plasma density fluctuations and their first-order differences in the structural turbulence observed in the hightemperature plasma of L-2M, TJ-II, and LHD [3] vary substantially in time. Heavy tails in this case can arise as a result of a certain influence function (with memory) or as a result of a great number of events with abnormally large amplitudes in first-order differences [11]. Samples of firstorder differences of such processes are usually independent and homogeneous. The adequacy of the proposed models is confirmed by the results of statistical analysis giving a good fit of mixtures of normal distributions with real data. In our analysis we apply a well-developed technique of statistical separation of finite normal mixtures, in particular, the Estimation Maximization (EM) algorithm.

In this paper we consider the statistical characteristics of time samples of amplitudes and their first-order differences of the core plasma density fluctuations in stellarators LHD, L-2M and TJ-II and relation of these characteristics with the non- Brownian particles motion.

\section{Experimental results}

The parameters of the L-2M, LHD and TJ-II devices are described in detail in [3]. Density fluctuations in hightemperature plasma at the middle radius in TJ-II were studied by 2 -mm scattering technique $\left(k=3 \mathrm{~cm}^{-1}\right.$ and $\left.k=6 \mathrm{~cm}^{-1}\right)$. Plasma density fluctuations in the heating region near the axis of the plasma column in L-2M $\left(k=20 \mathrm{~cm}^{-1}\right.$ and $\left.k=40 \mathrm{~cm}^{-1}\right)$ and LHD $\left(k \sim 30 \mathrm{~cm}^{-1}\right)$ were measured from scattered radiation of the heating gyrotron [12]. The correlation and statistical characteristics of time samples of amplitudes of density fluctuations and their first-order differences are analyzed. The first-order difference of amplitudes of plasma density fluctuations in a time sample is defined as $\Delta X_{j}=X_{j}\left(t_{j}\right)$ $-X_{j-1}\left(t_{j-1}\right)$ [7]. The first-order differences of the random value $\Delta X_{j}$ depend on the linear and nonlinear growth and decay processes involved in the formation of plasma density fluctuations under conditions of a particular experiment.

Figure 1 shows the autocorrelation functions (ACFs) of amplitudes of plasma density fluctuations and their first-order
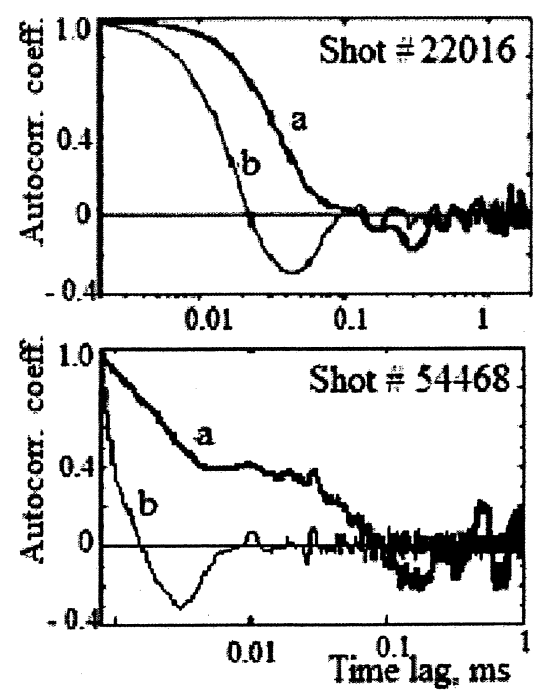

Fig. 1 Autocorrelation functions of amplitudes of density fluctuations (a) and their first-order differences (b) in LHD (the upper plot) and L-2M (the lower plot).

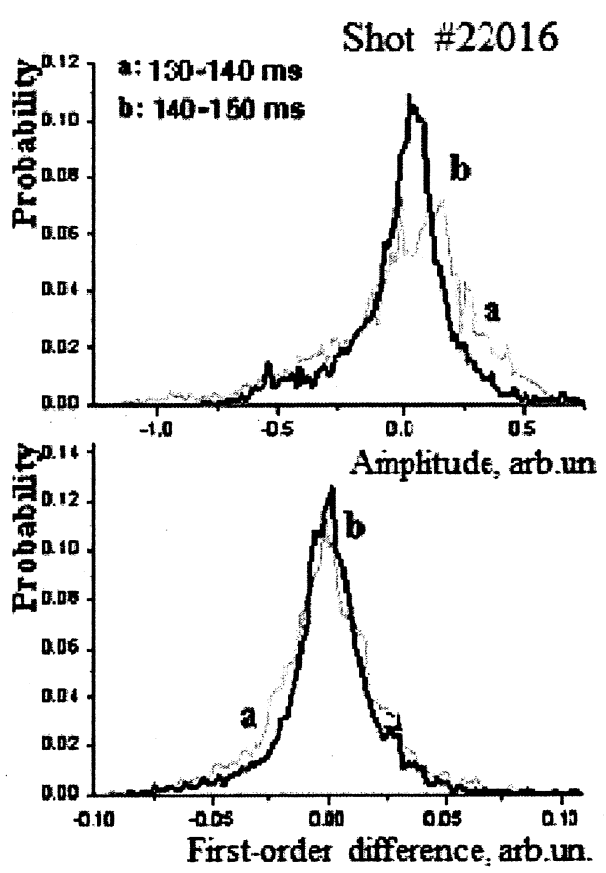

Fig. 2 Probability densities of amplitudes of fluctuations (the upper plot) and their first-order differences (the lower plot) in successive time intervals in LHD. 
differences in L-2M $\left(k=40 \mathrm{~cm}^{-1}\right)$ and LHD $\left(k \sim 30 \mathrm{~cm}^{-1}\right)$. It is well known that, for the structural plasma turbulence, the time sample is not homogeneous and independent (the ACF demonstrates the presence of a long-lived component). The independence of the first-order differences in the structural plasma turbulence is evidenced by the absence of long-term correlations in their ACF.

The scale mixtures of Gaussian laws model experimental probability density functions of the first-order differences. As an illustration, we only present PDF figures of strong structural turbulence in LHD, similar results were obtained in other two devices.

In Fig. 2, one can see the PDFs of time samples of fluctuation amplitudes measured in successive time intervals during an LHD discharge. Note that a non-Gaussian PDF of fluctuation amplitudes, which is typical in the structural plasma turbulence, varies noticeably during the discharge (the upper plot in Fig. 2). Unlike the PDF of the amplitudes, the PDF of the first-order differences in the steady-state discharge varies only slightly (the lower plot in Fig. 2). The independence of the time samples of the first-order differences and the stability of their PDF suggest that subordinated Lèvy processes can model the structural plasma turbulence. Below, it will be shown that mixtures of normal distributions give a good fit to the PDF of the first-order differences of the fluctuation amplitudes in the structural plasma turbulence.

The longest time samples of fluctuations up to $200 \mathrm{~ms}$ were measured in TJ-II, where measurements were made at the middle radius (far from the heating region and the plasma boundary). The analysis of samples (histograms) of the firstorder differences of the plasma density fluctuations demonstrated the dependence of the fitted distribution on the sample size (the length of the corresponding time series). The histograms for relatively short time samples $\left(10^{3}\right.$ points, which corresponds to the interval of $2 \mathrm{~ms}$ ) are well described by mixture of two Gaussian distributions

$$
\begin{aligned}
& f\left(x ; p, \mu_{1}, \mu_{2}, \sigma_{1}, \sigma_{2}\right)=\frac{p}{\sqrt{2 \pi} \sigma_{1}} \exp -\frac{\left(x-\mu_{1}\right)^{2}}{2 \sigma_{1}^{2}}+ \\
& \frac{1-p}{\sqrt{2 \pi} \sigma_{2}} \exp \left[-\frac{\left(x-\mu_{2}\right)^{2}}{2 \sigma_{2}^{2}}\right],
\end{aligned}
$$

$p, 1-p$ are probabilities (weights), $\mu_{1}, \mu_{2}$ are drift rates and $\sigma_{1}, \sigma_{2}>1$ are diffusion rates of these two distributions. The coincidence of the model with experimental data was tested by Kolmogorov-Smirnov goodness-of-fit test and reached $P$ $=0.9$ for equal intervals throughout discharge duration $(P$ is the test characteristic which means the probability to attain worse coincidence in the next experiment as compared to the observed one: the closer $P$ to 1 , the better the attained coincidence; the closer $P$ to 0 , the worse the coincidence; see, e.g., [13]). For the longer samples up to $3 \times 10^{4}$ points, a mixture of three Gaussian distributions

$$
\begin{aligned}
& f\left(x ; p, \mu_{1}, \mu_{2}, \mu_{3}, \sigma_{1}, \sigma_{2}, \sigma_{3}\right) \\
& =\sum_{k=1}^{3} \frac{p_{k}}{\sqrt{2 \pi} \sigma_{k}} \exp \left[-\frac{\left(x-\mu_{k}\right)^{2}}{2 \sigma_{k}^{2}}\right]
\end{aligned}
$$

$\left(p_{k}>0, p_{1}+p_{2}+p_{3}=1\right)$ provides good fit with experimental data $(P \approx 0.8)$.

The length of stationary time samples of plasma density fluctuations in the heating region of LHD did not exceed $5 \cdot 10^{3}$ points. Figure 3 shows the histogram of a time sample consisting of 2,000 points. In the same figure one can see a model normal distribution which fails to agree with

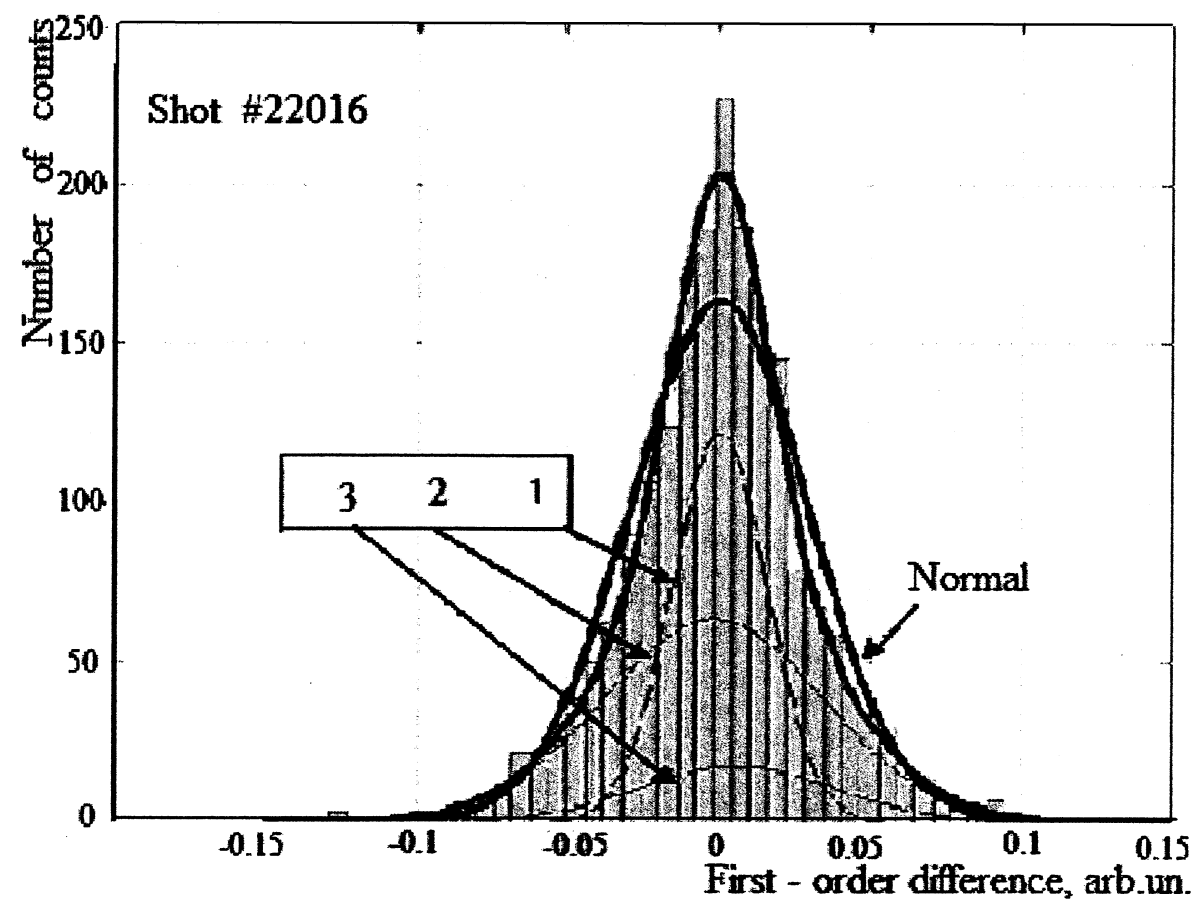

Fig. 3 Modeling of the PDF of a time sample of first-order difference of fluctuations in LHD by mixing three Gaussian distributions, labeled by 1,2 and 3 , respectively. 
experimental data: the Kolmogorov-Smirnov test for fitting one normal distribution with parameters $\mu=-0.000148, \sigma=$ 0.029439 gives a significance value $P=0.0017$. This time sample is modeled by a mixture of three Gaussian processes with weights $p_{1}=0.482032,\left(\mu_{1}=-0.001758, \sigma_{1}=0.036352\right)$; $p_{2}=0.400771\left(\mu_{2}=-0.000051, \sigma_{2}=0.015801\right) ; p_{3}=$ $0.117197\left(\mu_{3}=0.006141, \sigma_{3}=0.032432\right)$. The KolmogorovSmirnov test proves that this model mixture fits process adequately: $P=0.8176$. The probability density function for this mixture is also shown in the figure. The representation of the PDF of first-order differences of fluctuation amplitudes by scale mixtures of normal processes corresponds to the Lèvy process, which allows one to estimate the relative contribution from different turbulent diffusion mechanisms to transport processes as well as diffusion coefficients themselves.

\section{Conclusions}

From this analysis we make three definite conclusions: (i) the normal model fails to fit the PDF of first-order differences of fluctuations in the structural plasma turbulence with a very high significance level; (ii) even two-component normal mixtures demonstrate very good fit with very high $P$ value for rather short samples of fluctuations; (iii) for longer samples, a slight complication of the model (introduction of the third component) practically solves the problem.

The main conclusion is that the registered process can be successfully modeled by a combination of a finite number of diffusive processes each of which corresponds to a certain diffusive mechanism related to the structural turbulence in a plasma transport process. This combination is a subordinated Lèvy process (more precisely, a subordinated Wiener process) with a subordinator having a discrete distribution. As this is so, the characteristics of the combined process (the shares of each diffusion component as well as their drift and diffusive rates) can be estimated automatically, say, by the EM algorithm.
This work was supported in part by the Russian Foundation for Basic Research (project nos. 03-02-17269, 0402-16571, 02-01-01080a, 02-01-00949a) and the LIME Program (Japan).

\section{References}

[1] 30th EPS, St. Petersburg, 2003. http://eps2003.ioffe.ru/ public

[2] G.M. Batanov, V.E. Bening, V.Yu. Korolev et al., JETP Lett., 78, 502 (2003).

[3] G.M. Batanov, L.V. Kolik, A.E. Petrov et al., Plasma Phys. Rep. 29, 363 (2003).

[4] N.K. Kharchev, N.N. Skvortsova, K.A. Sarksyan, J. Math. Sci. 106, 2691 (2001).

[5] G.M. Batanov, A.E. Petrov, K.A. Sarksyan et al., JETP Lett. 67, 662 (1998).

[6] K.A. Sarksyan, N.N. Skvortsova, N.K. Kharchev and B.F. Milligen, Plasma Phys. Rep. 25, 312 (1999).

[7] G.M. Batanov, V.E. Bening, V.Yu. Korolev et al., JETP Lett. 73, 174 (2001).

[8] N.N. Skvortsova, K.A. Sarksyan and N.K. Kharchev, JETP Lett. 70, 201 (1999).

[9] J.T. Bendler and M.F. Shlessinger, The Wonderful World of Statistics (North-Holland, Amsterdam, 1985).

[10] B.V. Gnedenko and V. Yu. Korolev, Random Summation: Limit Theorems and Applications (CRC Press, Boca Raton, Fl, 1996).

[11] V.Yu. Korolev, Stereotype of normal laws and mechanism of genesis of distributions with heavy tails in Stochastic Models of Structural Plasma Turbulence (MAKS Press, Moscow, 2003) in Russian.

[12] N.N. Skvortsova, G.M. Batanov et al., J. Plasma Fusion Res. SERIES 5, 328 (2002).

[13] W.H. Press, B.P. Flannery, S.A. Teukolsky, W.T. Vetterling, Numerical Recipes in Pascal (Cambridge University Press, 1989). 